\title{
Comparación de la formación de carga eléctrica en aislamientos de XLPE con antioxidantes sólido y líquido mediante la técnica TSDC
}

\section{(Comparison of the formation of electric charge in XLPE insulation with solid and liquid antioxidants by TSDC technique)}

\author{
Idalberto Tamayo Ávila1, Ivanna Mishell Guaman Tumaille², Yandi Fernández Ochoa \\ Nilo Olegario Ortega Soliz ${ }^{4}$
}

\begin{abstract}
Resumen:
En este trabajo se comparó la presencia de carga eléctrica en dos aislamientos de polietileno reticulado (XLPE) de cables de media tensión mediante la técnica de las Corrientes de Despolarización Estimuladas Térmicamente (TSDC). A uno de los aislamientos se le añadió antioxidante en estado sólido y al otro en estado líquido. Hemos comprobado mediante el análisis de las medidas TSDC que el uso de antioxidante líquido es mejor puesto que el sólido crea defectos que actúan como centros de atrapamiento de la carga de espacio. En estos centros se acumula la carga ionizada en el aislante por la influencia combinada de la temperatura y el campo eléctrico además de la carga inyectada desde los electrodos semiconductores por el efecto de campos elevados.
\end{abstract}

Palabras clave: carga de espacio; polietileno reticulado (XLPE); antioxidante; TSDC

\begin{abstract}
:
In this paper the presence of electric charge in two insulation of cross-linked polyethylene (XLPE) in medium voltage cables was compared using the technique of Thermally Stimulated Depolarization Currents (TSDC). Antioxidant in solid form was added to one of the insulation, and in liquid form to the other. By analyzing the TSDC measures we verified that the use of liquid antioxidant is better because the solid antioxidant creates defects that act as centers for trapping the space charge. In these centers the ionized charge, by the combined effect of temperature and electric field, is accumulated in the insulation, in addition to the charge injected from the semiconductor electrodes by the effect of high fields.
\end{abstract}

Keywords: space charge; cross-linked polyethylene (XLPE); antioxidant; TSDC

\footnotetext{
1 Universidad Tecnológica Equinoccial, Facultad de Ciencias de la Ingeniería, Campus Occidental, Quito Ecuador (tai7012891@ute.edu.ec)

2 Universidad Tecnológica Equinoccial, Facultad de Ciencias de la Ingeniería, Campus Occidental, Quito Ecuador (gtim103597@ute.edu.ec)

${ }^{3}$ Universidad Tecnológica Equinoccial, Facultad de Ciencias de la Ingeniería, Campus Santo Domingo, Quito Ecuador (yandi.fernandez@ute.edu.ec)

${ }^{4}$ Universidad Tecnológica Equinoccial, Facultad de Ciencias de la Ingeniería, Campus Santo Domingo, Quito Ecuador (nilo.ortega@ute.edu.ec)
} 


\section{Introducción}

El polietileno reticulado XLPE es un polímero de cadena cruzada que se emplea como aislamiento en cables de media tensión (Figura 1) el mismo se obtiene en una reacción de reticulado durante un proceso de extrusión en el que se usa polietileno de baja densidad LDPE al que se le añade un determinado tipo de peróxido y antioxidante. Estos cables durante su funcionamiento están sometidos hasta tensiones de unos $20 \mathrm{kV}$ y pueden alcanzar la temperatura de $90{ }^{\circ} \mathrm{C}$. La función del aislamiento (c) de $5 \mathrm{~mm}$ de espesor es impedir el paso de carga eléctrica a través de él lo que garantiza un largo tiempo de servicio del cable en sus aplicaciones eléctricas por lo que debe estar diseñado de manera que la carga acumulada en todo su volumen sea mínima.

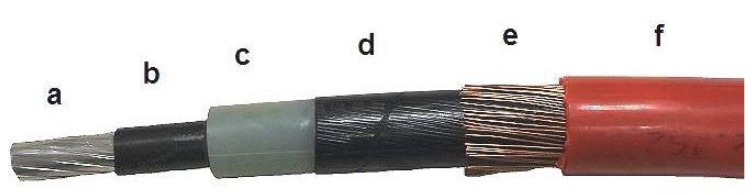

Figura 1. Cable de media tensión, aislamiento de XLPE (c).

El estudio de la formación de la carga de espacio en materiales aislantes sometidos a campos eléctricos es de especial interés puesto que su presencia influye en los mecanismos de conducción eléctrica del material, en el incremento del campo eléctrico local y en el proceso de ruptura dieléctrica que limita el tiempo de vida útil del aislante. La presencia de este tipo de carga en el XLPE de cables está influenciada por la oxidación del material por efecto de la temperatura (Frutos et al., 2007), los antioxidantes empleados (Sekii, Taya, \& Maeno, 2006), los subproductos de la reacción de reticulado (Maeno et al., 2005).

En trabajos anteriores ha quedado demostrado que durante el proceso de extrusión, en el que se alcanzan temperaturas de unos $300^{\circ} \mathrm{C}$, los electrodos semiconductores (b) y (d) difunden componentes químicas hacia el volumen del XLPE. También el tratamiento térmico posterior de las muestras provoca que continúen difundiéndose estas componentes desde las capas semiconductoras hacia el volumen y que penetren aún más hacia el interior del aislamiento. Estas componentes se disocian e ionizan por efectos del campo eléctrico y la temperatura contribuyendo a la formación de carga eléctrica en el aislante (Tamayo et al., 2004), pero en si no es la única causa que genera carga puesto que la oxidación de los antioxidantes también liberan cargas en el material y a campos elevados paquetes de carga de espacio que se forman y propagan por el volumen del material contribuyendo a la conducción del aislante (Chouikhi, Boukhris, Belgaroui, \& Kallel, 2013).

En este trabajo nos proponemos como objetivo comprobar mediante la técnica TSDC si la carga acumulada en el volumen de dos muestras de cables originales cambia por el hecho de haber usado 
antioxidante (di-tert-butyl dicarbonate) en estado sólido y en estado líquido, por lo que mediremos la carga acumulada en dos aislamientos en los que sólo se ha cambiado el estado del antioxidante.

\section{Metodología}

Dentro de las técnicas de análisis para estudiar la carga de espacio se encuentra la técnica de las Corrientes de Despolarización Estimuladas Térmicamente (TSDC), el principio de la misma se esquematiza en la Figura 2 y se basa en aplicar un campo eléctrico a la muestra para polarizarla eléctricamente a una temperatura determinada que generalmente es superior a la temperatura ambiente, luego se congelan los mecanismos de polarización bajando la temperatura con el campo aplicado. Finalmente se despolariza la muestra elevando la temperatura controladamente, la despolarización da lugar a una corriente eléctrica que se puede registrar en función de la temperatura con un sistema de adquisición de datos.

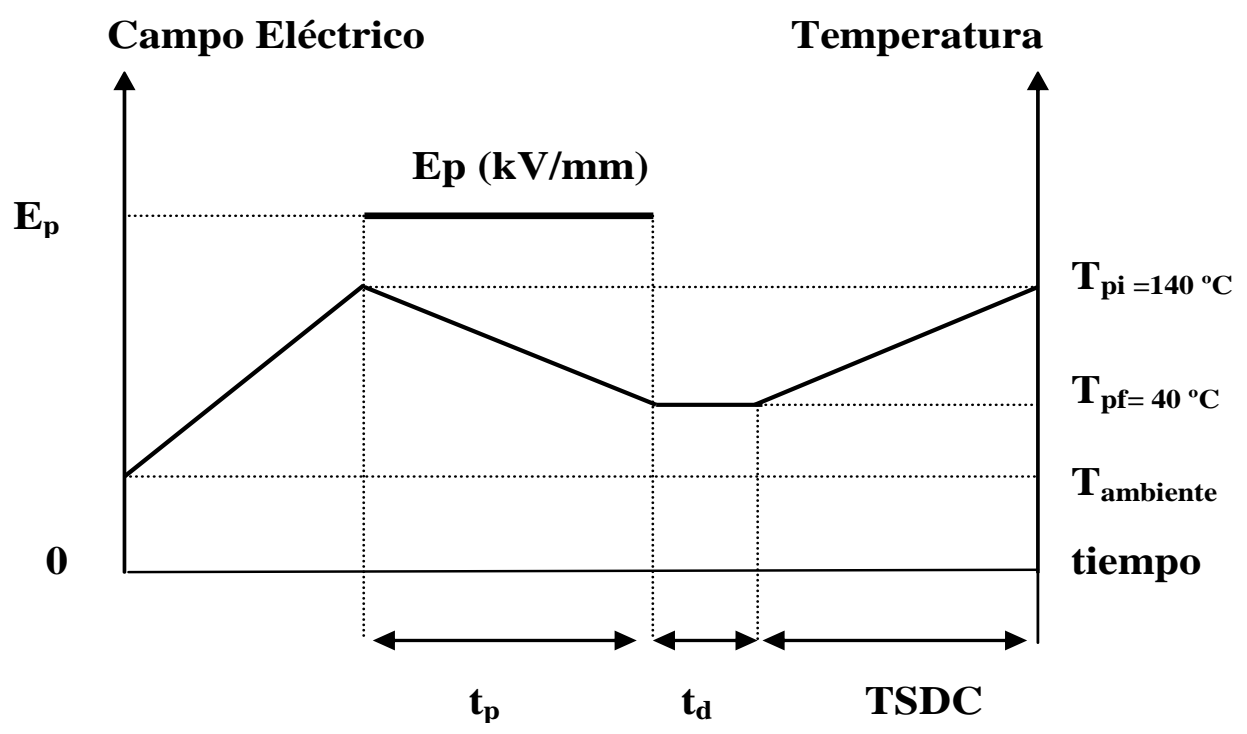

Figura 2. Etapas de Polarización y Despolarización.

La curva que se obtiene muestra la relajación de los mecanismos de polarización tantos dipolares como de carga de espacio. El área bajo la curva $i=f(T)$ es una medida de carga acumulada en el volumen del aislamiento y que interviene en la corriente de despolarización estimulada térmicamente (Figura 3). 


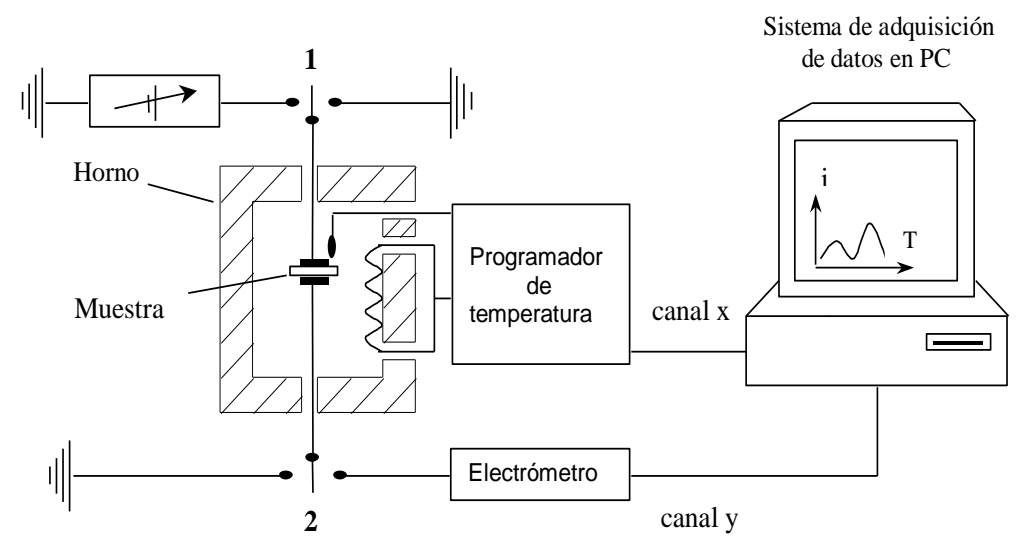

Figura 3. Esquema de la técnica TSDC

Las medidas TSDC fueron realizadas con la instalación del laboratorio DILAB de la Universidad Politécnica de Catalunya (Figura 4) en la que (1) es un horno en el que en su interior se encuentra la célula de medidas donde se ubican las muestras y se le aplica el campo eléctrico polarizador con una fuente de alta tensión (2). Con la instalación se pueden efectuar rampas controladamente con un programador de temperaturas (3). La corriente se mide con el electrómetro (4) en el orden de los pA. En un PC con sistema de adquisición (5) queda registrada la curva de corriente en función de la temperatura. La instalación consta además de un sistema de enfriamiento (6) que permite efectuar ciclos de calentamiento y enfriamiento a temperatura controlada.

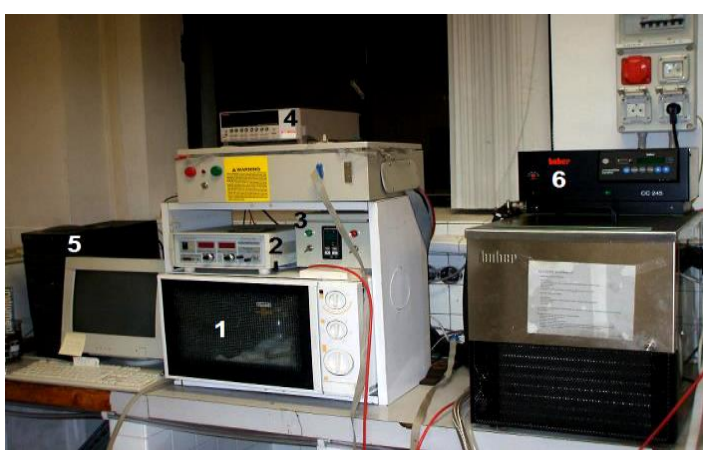

Figura 4. Instalación Experimental para las medidas TSDC.

Se midieron dos tipos de muestras de cables una a las que se le añadió antioxidante en estado sólido y otra en estado líquido. Las mismas fueron calentadas a una velocidad de $2{ }^{\circ} \mathrm{C} / \mathrm{min}$ desde temperatura ambiente hasta $140{ }^{\circ} \mathrm{C}$, a esta temperatura se comenzó a aplicar un campo polarizador de $4 \mathrm{kV} / \mathrm{mm}$ mientras se enfriaba a velocidad controlada desde de $140{ }^{\circ} \mathrm{C}$ hasta $40{ }^{\circ} \mathrm{C}$ donde se desconectó el campo y se procedió a calentar nuevamente la muestra a la misma velocidad hasta $140{ }^{\circ} \mathrm{C}$ registrándose la corriente de despolarización en función de la temperatura como se esquematiza en la Figura 3. 


\section{Resultados}

En la Figura 5 y Figura 6 mostramos las curvas TSDC para las dos muestras estudiadas, repetimos las medidas 5 veces y vemos como inicialmente a la que se le añadió antioxidante sólido se obtiene menos carga, nótese que el área bajo cada curva comienza a disminuir cuando se comienzan a repetir los ciclos de temperatura propios de la aplicación de la técnica TSDC. El área calculada en el caso del antioxidante sólido da una carga de 10,64 nC y va disminuyendo hasta 6,28 nC cuando se han realizado 5 descargas. En la muestra con antioxidante líquido la carga inicial es de 16, 53 nC y va disminuyendo hasta 10, 52 nC acumulando mayor carga que en el caso del antioxidante sólido.

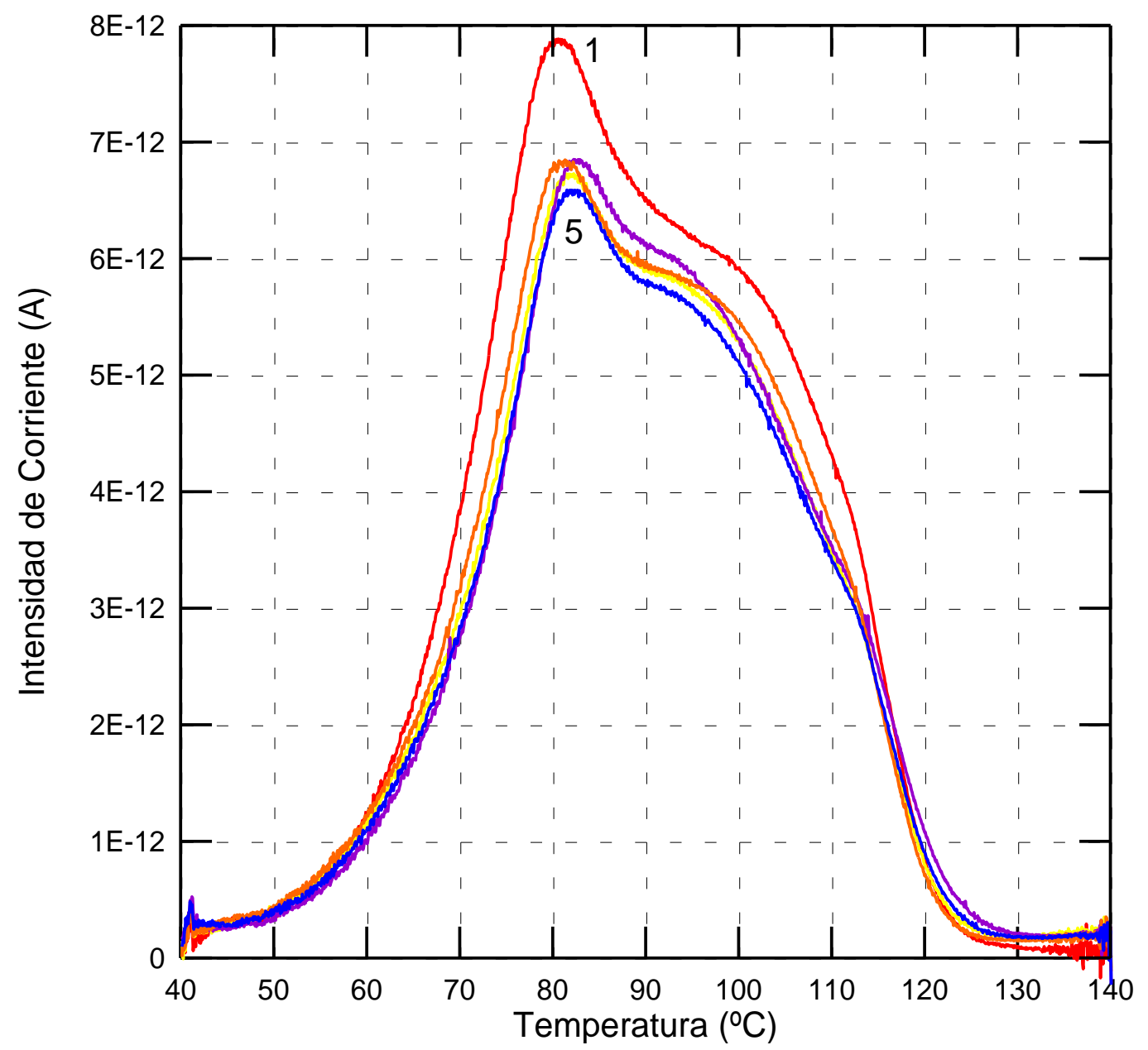

Figura 5. Medidas TSDC en muestra de cable con antioxidante sólido. 


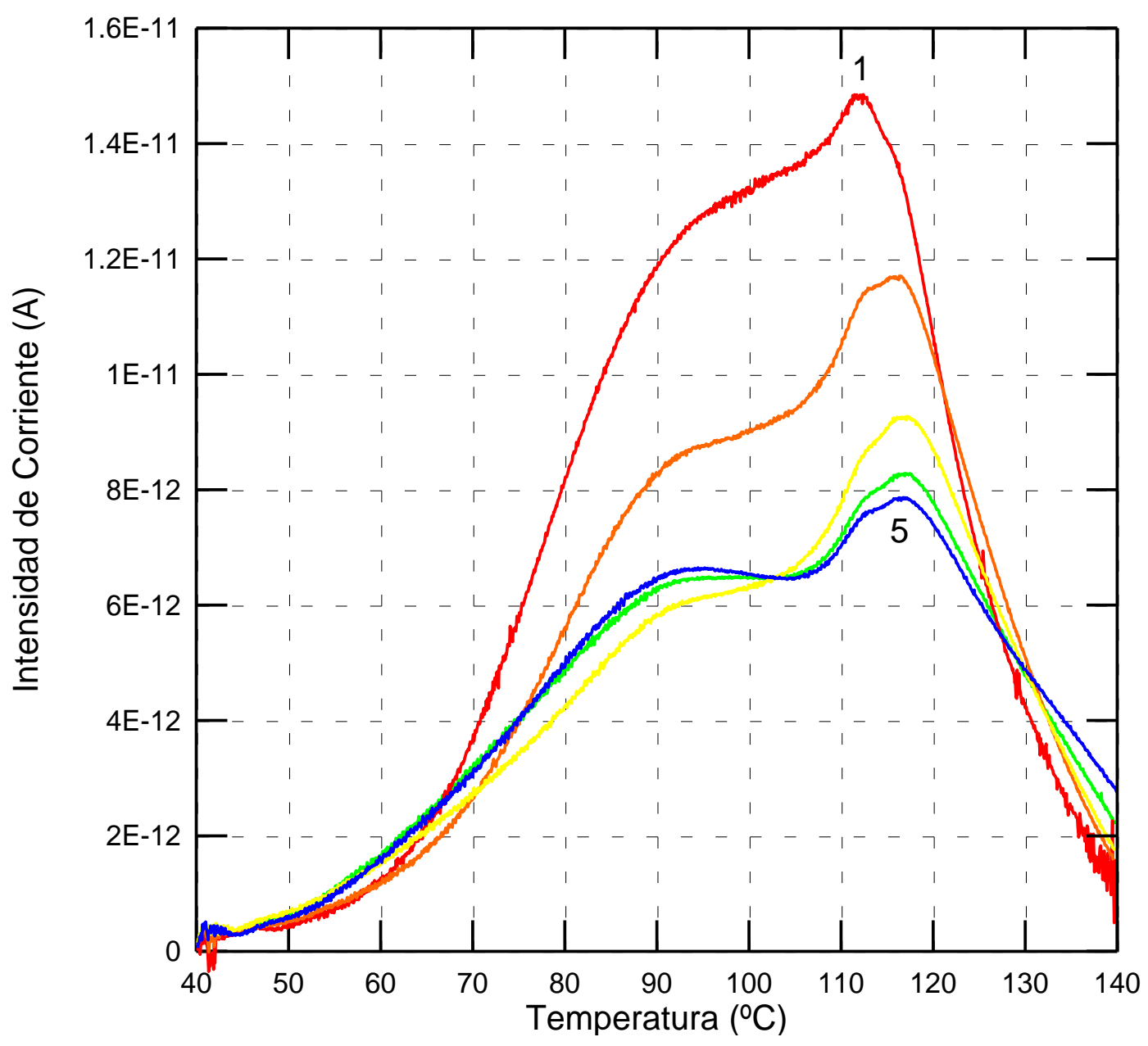

Figura 6. Medidas TSDC en muestra de cable con antioxidante líquido.

En la Tabla 1 resumimos las cargas calculadas para cada una de las muestras al repetir la descarga TSDC cinco veces con las mismas condiciones. Nótese que la carga disminuye y como la muestra con antioxidante líquido acumula más carga.

Tabla 1. Cargas calculadas para cada muestra en cinco repeticiones.

\begin{tabular}{|c|c|c|}
\hline $\begin{array}{c}\text { Descarga } \\
\text { TSDC }\end{array}$ & $\begin{array}{c}\text { Antioxidante Sólido } \\
\text { Carga (nC) }\end{array}$ & $\begin{array}{c}\text { Antioxidante Líquido } \\
\text { Carga (nC) }\end{array}$ \\
\hline 1 & 10,64 & 16,53 \\
\hline 2 & 6,63 & 13,05 \\
\hline 3 & 6,46 & 10,64 \\
\hline 4 & 6,45 & 10,62 \\
\hline 5 & 6,28 & 10,52 \\
\hline
\end{tabular}


Hasta aquí queda claro que los ciclos de temperatura provocan una disminución de la carga en ambas muestras y para observar más detenidamente el comportamiento con la temperatura procedimos a realizar otro tipo de experimento que consistió en tratar térmicamente a $140^{\circ} \mathrm{C}$ durante varias horas a ambas muestras para observar el efecto sobre la acumulación carga con el tiempo del tratamiento y también al aplicar un campo polarizador mucho más grande de unos $20 \mathrm{kV} / \mathrm{mm}$.

En la Figura 7 cuando las muestras están si tratar térmicamente se obtienen descargas con corriente negativa de 40 a $72{ }^{\circ} \mathrm{C}$. La corriente negativa es propia de la relajación de carga inyectada desde los electrodos a altos campos eléctricos, las áreas calculadas muestran que en el caso del antioxidante sólido es mayor $(-2,63 \mathrm{nC})$ que en el líquido $(-0,587 \mathrm{nC})$. A partir de $72{ }^{\circ} \mathrm{C}$ se obtiene una corriente de descarga positiva siendo mayor la carga en el caso del antioxidante líquido $(12,146 \mathrm{nC})$ que en el caso del sólido $(8,514 \mathrm{nC})$.

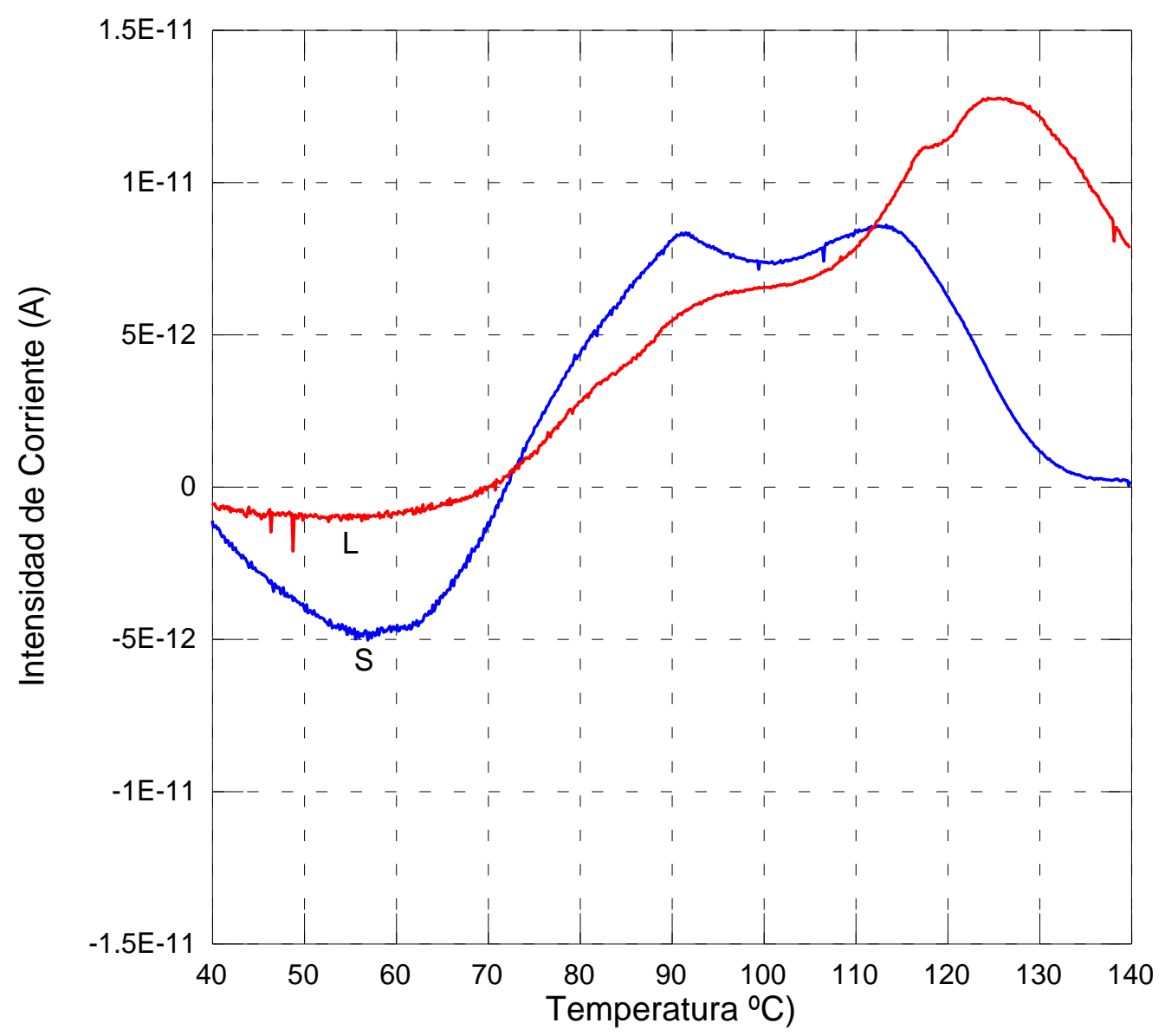

Figura 7. Medidas TSDC a $20 \mathrm{kV} / \mathrm{mm}$ en muestra con antioxidante sólido (S) y en muestra con antioxidante líquido $(\mathrm{L})$, ambas sin tratamiento térmico. 
En la Figura 8 cuando se trataron térmicamente las muestras durante 8 horas a $140^{\circ} \mathrm{C}$ se obtienen resultados entre $40-95{ }^{\circ} \mathrm{C}$ que indican a una fuerte inyección de carga desde los electrodos que es más notable en la muestra con antioxidante sólido $(-14,084 \mathrm{nC})$ que en la que contiene antioxidante líquido $(-3,419 \mathrm{nC})$. A partir de los $95{ }^{\circ} \mathrm{C}$ se nota también más carga en el caso del antioxidante sólido $(4,697 \mathrm{nC})$ que en el líquido $(-0,249 \mathrm{nC})$.

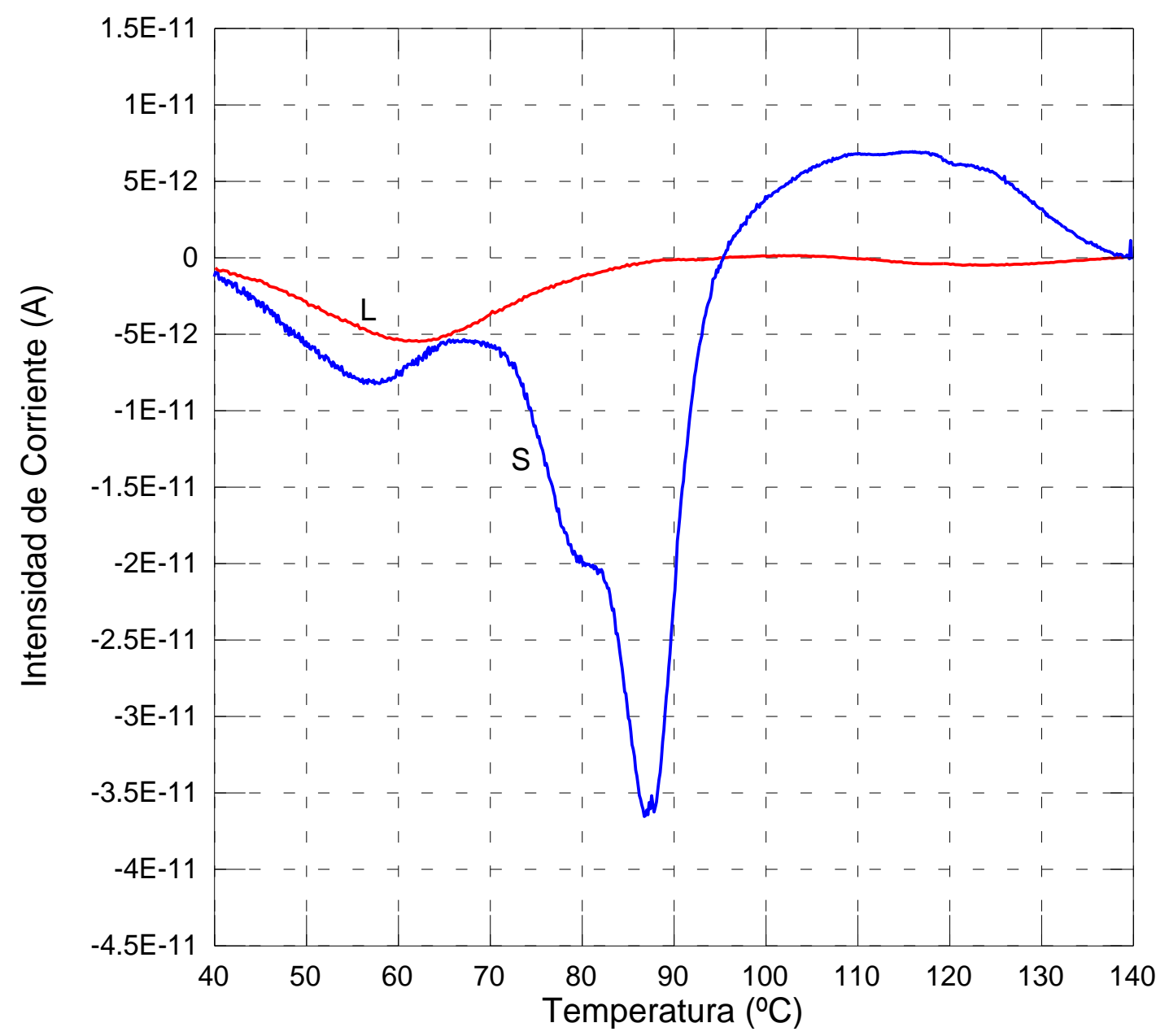

Figura 8. Medidas TSDC a $100 \mathrm{kV}$ en muestra con antioxidante sólido (S) y en muestra con antioxidante líquido $(\mathrm{L})$, ambas con tratamiento térmico de $8 \mathrm{~h}$ a $140^{\circ} \mathrm{C}$

En la Figura 9 se observa que en el caso de las muestras tratadas durante una semana a $140^{\circ} \mathrm{C}$, la que contiene antioxidante líquido acumula mucho menos carga $(1,196 \mathrm{nC})$ si se compara con la de antioxidante sólido $(6,456 \mathrm{nC})$. 


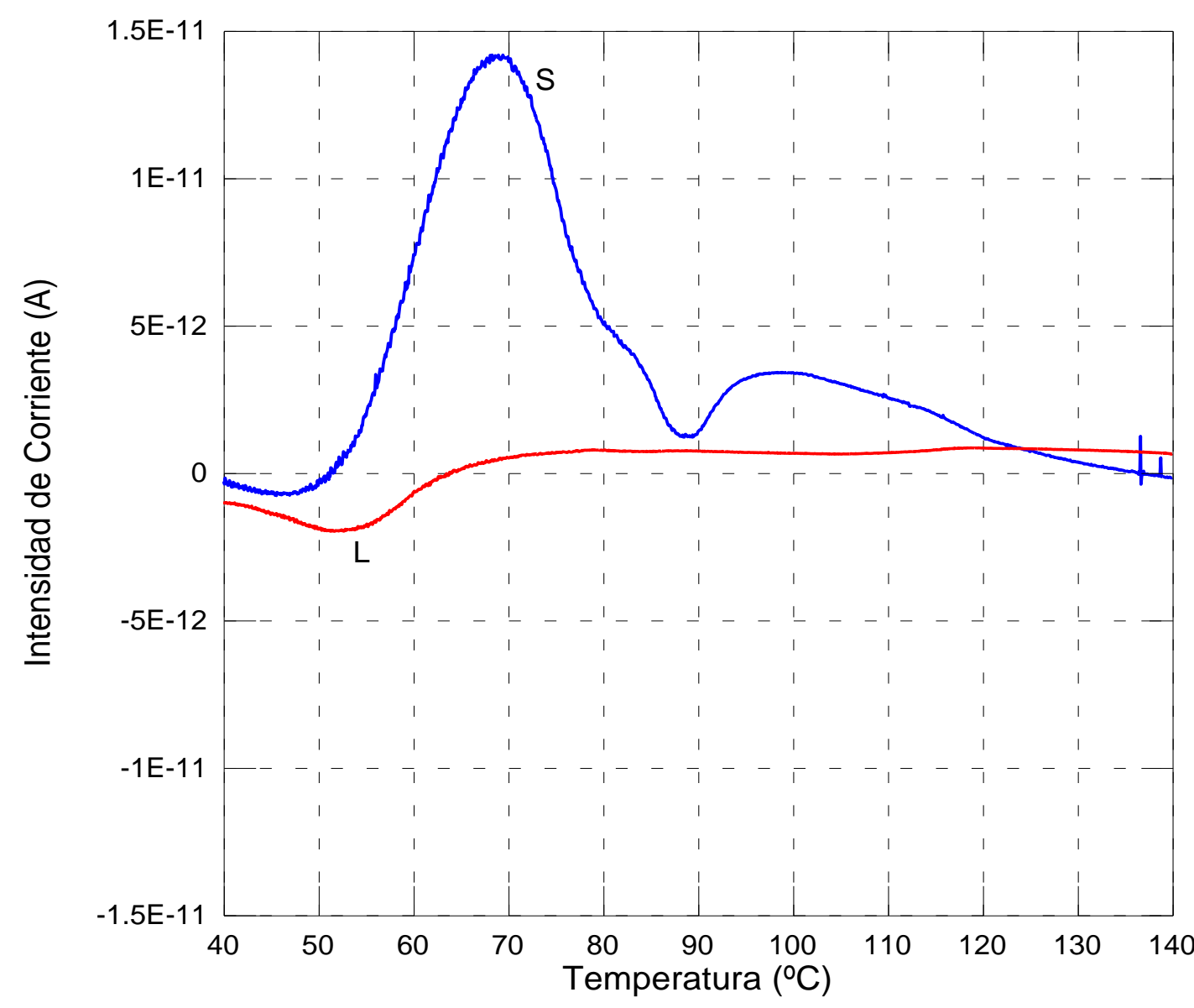

Figura 9. Medidas TSDC a $100 \mathrm{kV}$ en muestra con antioxidante sólido (S) y en muestra con antioxidante líquido $(\mathrm{L})$, ambas con tratamiento térmico de $168 \mathrm{~h}$ a $140^{\circ} \mathrm{C}$

\section{Discusión}

El polietileno reticulado con antioxidante en estado líquido después de someterse a un tratamiento térmico a la temperatura de $140{ }^{\circ} \mathrm{C}$ disminuye la cantidad de carga eléctrica acumulada en su volumen si se compara con el polietileno con antioxidante sólido. Este comportamiento puede ser debido a que el tratamiento térmico de las muestras con antioxidante líquido a la temperatura tratada provoca una fácil evaporación, disociación e ionización dando lugar que las componentes volátiles de los subproductos de la reacción de reticulado y que generalmente se acumulan en las interfaces, salgan hacia al exterior, razón por la cual contribuye finalmente a que haya menos carga acumulada.

El mayor número de defectos en el volumen del XLPE causado por las partículas de antioxidante en estado sólido provoca que estos se comporten como centros de atrapamiento de carga y que haya mayor acumulación en el volumen del aislamiento. La oxidación del antioxidante por efecto de la temperatura también contribuye a que finalmente se acumule más carga en el XLPE con antioxidante sólido. 


\section{Conclusiones y Recomendaciones}

En antioxidante en estado líquido genera mayor cantidad de carga en el XLPE de las muestras de los cables sin tratar térmicamente mientras que en estado sólido causa más carga en el volumen del XLPE si se tratan las muestras a $140{ }^{\circ} \mathrm{C}$ previamente durante horas. Puesto que estos cables pueden alcanzar temperaturas en cortocircuito de hasta unos $250^{\circ} \mathrm{C}$ consideramos que la utilización del antioxidante en estado líquido combinado con un tratamiento térmico del cable es más apropiado que el antioxidante sólido porque provoca menos defectos estructurales en el XLPE, una fácil desgasificación por efecto de la temperatura y acumula menos carga eléctrica. Se sugiere un estudio de la microestructura y morfología en ambos aislamientos antes y después del tratamiento térmico.

\section{Bibliografía}

Chouikhi, S., Boukhris, I., Belgaroui, E., \& Kallel, A. (2013). Space charge packets in polyethylene nano-scales under dc apllied voltage. Journal of Electrostatics 71, 14-20.

Frutos, F., Acedo, M., Mudarra, M., Belana, J., Orrit, J., Diego, J. A., \& Sellares, J. (2007). Effect of annealing on conductivity in XLPE mid-voltage cable insulation. Journal of Electrostatics. Volumen 65 Issue 2, 122-131.

Maeno, Y., Hirai, N., Ohki, Y., Tanaka, T., Okashita, M., \& Maeno, T. (2005). Effect of Cross-linking Byproducts on Space Charge Formation in Cross-linked Polyethylene. IEEE Trans. Dielectr. Electr. Insul. Vol. 12, 90-97.

Sekii, Y., Taya, A., \& Maeno, T. (2006). Effect of Antioxidants on Space Charge Generation in Crosslinked Polyethylene and EPR. IEEE Conf. Electr. Insul. Dielectr. Phenomena (CEIDP) (págs. 133-137). IEEE.

Tamayo, I., Belana, J., Diego, J. A., Cañadas, J. C., Mudarra, M., \& Sellares, J. (2004). Space Charge Studies of Crosslinked Polyethylene Midvoltage Cable Insulation by Thermally Stimulated Depolarization Current, Infrared/Fourier Transform Infrared, and Scanning Electron Microscopy. Journal of Polymer Science: Part B: Polymer Phisics. Vol 42, 4164-4174. 